www.revistadyo.com

\title{
Agilidad, flexibilidad de producción e innovación en la empresa manufacturera espa- ñola
}

\author{
Angel Martínez Sánchez*, Manuela Pérez Pérez y Silvia Vicente Oliva
}

Recibido: 2 de Febrero de 2018 / Aceptado: 26 de Febrero de 2018

\section{Resumen}

Este artículo analiza en una muestra de empresas manufactureras españolas la relación de la producción ágil con el esfuerzo interno y la cooperación externa en innovación tecnológica. Los resultados del estudio indican que las empresas con mayor capacidad de agilidad utilizan con más intensidad un amplio conjunto de tecnologías agiles de desarrollo, fabricación y suministro. Las empresas más ágiles también cooperan externamente más en innovación y dicha cooperación modera la flexibilidad de producción de las empresas, indicando con ello que, gracias a la cooperación externa en innovación las empresas con menor flexibilidad de producción pueden aun así conseguir mayor capacidad de agilidad.

\section{Palabras clave}

Producción Ágil. Innovación. Cooperación Tecnológica. Capacidad de Absorción del Conocimiento. Cadena de suministro.

\section{Introducción}

Las empresas compiten en mercados que son cada vez más globalizados y cambiantes. Este tipo de competencia requiere de capacidades que mejoren la eficiencia, calidad e innovación de las empresas pero también de capacidades de cambio y adaptación a las modificaciones del entorno. Las empresas han de ser por tanto flexibles e innovadoras para poder ofrecer nuevos productos en respuesta a los cambios impredecibles en el mercado o anticipándose incluso a los mismos. La flexibilidad de producción ha constituido un objetivo clásico de la estrategia de producción de las empresas. Si bien el concepto y medición de la flexibilidad de producción estaba centrado en un principio en el área de Operaciones manufactureras (flexibilidad de fabricación) su ámbito de estudio fue ampliándose posteriormente a las áreas de recursos humanos (ya que la flexibilidad de los recursos humanos es una buena base sobre la que desarrollar otras dimensiones de flexibilidad empresarial) y de forma más reciente a la cadena de suministro porque son las cadenas de suministro quienes compiten actualmente entre sí más que las propias empresas de forma aislada.

En consecuencia la flexibilidad de producción como objetivo de producción en mercados dinámicos y hostiles ha quedado mayormente englobada bajo otro concepto, el de la producción ágil, paradigma de producción del siglo XXI. La flexibilidad ha devenido en una condición necesaria de la

Angel Martínez Sánchez

anmarzan@unizar.es

Departamento de Dirección y Organización de Empresas.

Escuela de Ingeniería y Arquitectura. María de Luna,

3.Universidad de Zaragoza 50018. Teléfono 876555024 agilidad pero no en un sinónimo de la misma. La producción ágil necesita de tecnologías flexibles y por ello la flexibilidad de producción es una de sus principales prioridades competitivas pero también necesita mano de obra muy cualificada y formada, así como de unos sistemas de información compartida que respondan con rapidez a los cambios continuos e impredecibles del mercado. Si bien la producción ágil no es ya un concepto emergente, pues como tal se remonta a la última década del siglo XX (Goldman y Nagel, 1991), no lo es tanto el estudio sistemático de su aplicación. Por ejemplo en España no existen muchos trabajos que hayan analizado la producción ágil con datos empíricos de empresas; Vázquez $\square$ Bustelo et al. (2007) realizaron un estudio pionero en el que evidenciaban que las empresas manufactureras españolas en entornos más dinámicos utilizaban con mayor intensidad las tecnologías ágiles (de diseño, producción y administración) y obtenían a su vez mejores resultados. Otros estudios internacionales también han analizado facilitadores y determinantes de la agilidad, pero en la actualidad y dado que la producción ágil es un enfoque integrado de producción y gestión empresarial, resulta más útil profundizar en la relación existente entre el núcleo central de la producción ágil y las otras capacidades de gestión que posibilitan la agilidad empresarial a través de los flujos de conocimiento.

En particular, un área poco desarrollada en el contexto de la producción ágil es el efecto de los esfuerzos internos y externos que hace la empresa para innovar y desarrollar su capacidad de agilidad. Las empresas ágiles han de ser innovadoras porque, por una parte, compiten en mercados cambiantes que requieren satisfacer a consumidores exigentes con mejores e innovadores productos. Por otra parte, el ritmo y la incertidumbre de dichos cambios obligan a las empresas a cooperar en innovación con otros agentes y a mejorar su capacidad de absorción de conocimientos externos. Estos esfuerzos de la empresa pueden influir no solamente en su 
resultado de innovación sino también en el desarrollo de las capacidades de flexibilidad de producción y agilidad. El principal objetivo de nuestro trabajo es por tanto contribuir con datos de empresas manufactureras españolas a analizar la relación de determinados componentes de la capacidad de absorción de conocimientos de la empresa con la capacidad de flexibilidad de producción y agilidad de le empresa. En particular pretendemos analizar el efecto moderador que la cooperación externa en innovación puede tener en la contribución de la flexibilidad de producción a la agilidad, así como el efecto moderador del apoyo organizativo a la innovación a la contribución de la capacidad de innovación a la agilidad. Tanto la cooperación externa como el apoyo organizativo interno constituyen elementos esenciales de la capacidad de absorción de conocimientos de la empresa, los cuales pueden mejorar el resultado de innovación que tan necesario es para que las empresas sean ágiles y competitivas. Nuestro artículo se estructura de la siguiente forma: la próxima sección plantea el modelo teórico de investigación y explica los principales conceptos utilizados en el trabajo; después se explica la metodología del estudio, seguido de los resultados empíricos y de su discusión para terminar con la conclusión y limitaciones del trabajo.

\section{Teoría e hipótesis}

\subsection{La perspectiva de recursos y la perspectiva de capacidades dinámicas}

Nuestro desarrollo teórico se apoya en la perspectiva de recursos y en la perspectiva de capacidades dinámicas, ya que ambas contribuyen a explicar las implicaciones competitivas de la producción ágil y de la innovación. Por una parte, la premisa básica de la perspectiva de recursos es que la ventaja competitiva de una empresa reside fundamentalmente en la aplicación de recursos que sean valiosos, escasos, difíciles de imitar y no sustituibles (Barney, 1991). La perspectiva de recursos ha recibido una atención considerable en el campo de la estrategia empresarial pero también de forma creciente en las áreas de producción e innovación.

Por otra parte, la perspectiva de las capacidades dinámicas considera que la acumulación de recursos no es suficiente para tener éxito. Las empresas necesitan capacidades para integrar, reconfigurar, desarrollar y aplicar dichos recursos (Teece et al., 1997). Aunque la innovación sea muy importante en sí misma para que las empresas compitan y mejoren sus resultados, la gestión de la innovación no es el único factor de éxito. Las empresas también necesitan capacidades dinámicas que les permitan crear, expandir o modificar sus bases de recursos (Kohlbacher, 2013). Las capacidades dinámicas contribuyen a la ventaja competitiva de las empresas posibilitando ventajas temporales que permiten a la empresa permanecer por delante de sus competidores (Teece, 2007). Por ello, la posesión o control de capacidades dinámicas que permiten por ejemplo la rápida reconfiguración de las Operaciones de una empresa representa un gran potencial, especialmente en entornos dinámicos y rápidamente cambiantes.

\subsection{La producción ágil}

El concepto de la producción ágil surgió a finales de la década de 1990, cuando las grandes empresas manufactureras se enfrentaron al reto de flexibilizar más sus Operaciones, con tiempos de producción más cortos y mayor variedad de productos. Aunque en la literatura existen varias definiciones de la producción ágil algunos autores como, por ejemplo, Gunasekaran y Yusuf (2002) las han resumido y analizado para destacar que la producción ágil se centra en la búsqueda de la competencia global respondiendo con rapidez a las demandas dinámicas de los clientes y fabricando productos con muchas variedades e innovaciones. Por tanto, una organización que evolucione hacia la producción ágil no solo ha de disponer de una capacidad flexible de producción sino también, entre otras, de una capacidad concurrente de diseño de nuevos productos y de desarrollo de procesos así como de una colaboración estratégica con proveedores y otros agentes de la cadena de suministro. La empresa ágil ha de ser una organización con mayores niveles de conocimiento, tanto a nivel de stock como de flujos dentro y fuera de la empresa. Un examen del concepto de la producción ágil a la luz de la perspectiva de las capacidades dinámicas ofrece dos importantes implicaciones: (1) que la agilidad es una capacidad que permite a la empresa responder a un entorno empresarial incierto y cambiante para mantener o mejorar su posición en el mercado, y (2) que la agilidad, como capacidad dinámica, implica la explotación de las capacidades internas y externas de la empresa, el desarrollo de otras nuevas y su renovación para responder a los cambios en el entorno empresarial.

Los estudios empíricos muestran que las empresas en entornos altamente dinámicos utilizan tecnologías ágiles con mayor intensidad y obtienen mejores resultados (Narasimhan et al., 2006; Vázquez $\square$ Bustelo et al., 2007). Los entornos ágiles requieren tecnologías que permitan a trabajadores y máquinas compartir información de forma eficiente y eficaz con el fin de responder con rapidez a las necesidades cambiantes del mercado. El uso de robots, sistemas de comunicación en tiempo real, etc., ha redefinido el concepto de producción ágil y facilitado su implantación práctica y por ello constituye un componente esencial de cualquier esquema conceptual de producción ágil (Dubey y Gunasekaran, 2015). Asimismo, el concepto de agilidad se ha extendido a la cadena de suministro de forma que dicha agilidad forma parte actualmente de la gestión con éxito de las cadenas de suministro junto con otras capacidades como el alineamiento o la adaptación de dichas cadenas (Lee, 2004). Sin em- 
bargo, dado que la producción ágil es un enfoque integrado de producción, los estudios actuales no deberían solo analizar sus facilitadores específicos sino sobre todo la relación existente entre el núcleo central de la producción ágil y las otras capacidades de gestión de producción, innovación y cooperación que posibilitan la agilidad empresarial a través de los flujos de conocimiento. En particular, los esfuerzos de innovación internos y externos de la empresa para desarrollar productos innovadores y mejorar procesos de producción pueden contribuir de forma significativa a mantener y mejorar la capacidad de agilidad de la empresa. Ello requiere incorporar algunos conceptos y variables procedentes de la capacidad de absorción de la empresa con el fin de introducirlos dentro de nuestro modelo de investigación.

\subsection{Innovación y capacidad de absorción de conocimientos}

Para innovar, las empresas deben invertir en recursos y saber gestionar los procesos de desarrollo tanto de forma interna como, cada vez más, contando con los esfuerzos de otras empresas en cooperación conjunta o a partir solo de sus resultados. Las empresas que innovan mejor son aquellas que tienen una capacidad interna de desarrollo pero que también son capaces de cooperar y asimilar el conocimiento externo. A partir del concepto de capacidad de absorción, desarrollado originalmente desarrollado por Cohen y Levinthal (1990), otros investigadores han desarrollado posteriormente una tipología de dimensiones relacionadas con la capacidad de la empresa para identificar, asimilar, transformar y explotar los conocimientos externos (véase por ejemplo Zahra y George, 2002). La capacidad de desarrollar conjuntamente y absorber conocimientos del exterior es una capacidad valiosa y difícil de imitar por los competidores en entornos ágiles ya que está condicionada fundamentalmente por la trayectoria y conocimientos previos de cada empresa. Mediante la cooperación externa en innovación, la asimilación de conocimientos externos y el apoyo a las actividades internas de innovación, las empresas son capaces de responder de una forma más efectiva y ágil a las necesidades cambiantes de los clientes con nuevos o mejores productos, a la par que pueden también mejorar sus procesos de producción (Dobrzykowski et al. 2015). Por ejemplo, en el marco de las cadenas de suministro, Martínez y Lahoz (2017) han evidenciado con datos de empresas españolas cómo la agilidad puede constituir un mecanismo explicativo de la contribución positiva de la capacidad de absorción a los resultados empresariales. Por ello, algunos de los conceptos y herramientas incluidas dentro de la capacidad de absorción de conocimientos de la empresa pueden resultar útiles para analizar efectos moderadores entre las capacidades de flexibilidad de producción, innovación y agilidad.

\subsection{Modelo e hipótesis de investigación}

Distintos estudios empíricos muestran que las empresas que necesitan mayor agilidad en sus sistemas de producción y suministro compiten en entornos muy complejos y dinámicos. Los sistemas de producción ágil comparten el uso de prácticas (trabajo en equipo, empleados polivalentes, etc.) y objetivos de producción (coste, calidad, etc.) con otros sistemas productivos pero evidencian normalmente un uso más intensivo de dichas prácticas (Narasimhan et al., 2006) y responden a una mayor complejidad derivada de cambios más continuos e impredecibles. Las empresas que evolucionan hacia la producción ágil necesitan de una capacidad flexible de producción pero también de una capacidad concurrente de diseño y desarrollo de nuevos productos y procesos así como de colaboraciones estratégicas con otros agentes de la cadena de suministro. Por tanto, las diferencias entre sistemas de producción han de centrarse más bien en otras prioridades competitivas; en particular los esfuerzos de innovación constituyen un área poco desarrollada en la literatura de la producción ágil. Las empresas agiles deben ser organizaciones con mayores niveles y flujos de conocimiento porque tienen que competir en entornos inciertos con más productos innovadores que se desarrollan en el marco de cadenas de suministro ágiles. Por tanto, las empresas con sistemas de producción ágiles deberían invertir más en innovación y cooperación tecnológica externa para desarrollar sus capacidades de agilidad y alcanzar los resultados propuestos.

Sin embargo, el propósito de nuestro trabajo no es demostrar que las empresas ágiles han de invertir más en innovación y cooperación tecnológica externa. Otros investigadores ya han evidenciado que las empresas ágiles responden más rápido a los consumidores e invierten más recursos en tecnologías y prácticas ágiles (Narasimhan et al., 2006; Gligor et al., 2015). Estas prácticas de producción ágil permiten reducir el tiempo de comercialización y aumentar el grado de novedad de las innovaciones de producto: por ejemplo, el prototipado rápido y las impresoras $3 \mathrm{D}$ incrementan el número de opciones que pueden probarse en un periodo más corto de tiempo, lo cual mejora la probabilidad de éxito del nuevo producto. Nuestra investigación se centra más bien en explorar las implicaciones relacionadas con este mayor esfuerzo innovador. Por ejemplo, las empresas ágiles necesitan invertir específicamente en la adaptación y mejora continua de los sistemas de producción para obtener productos más competitivos, más innovadores y de mayor calidad. La compra de tecnologías de producción flexible no es en sí misma una fuente de ventaja competitiva sostenible porque puede ser fácilmente imitada por los competidores; lo que hace de la producción una fuente de ventaja competitiva sostenible es la adaptación personalizada de los sistemas ágiles de producción. Por tanto, el desarrollo e implantación 
de tecnologías de producción flexible puede requerir del acceso a expertos tecnológicos externos a la hora de implantar o mejorar la línea de producción.

Otra implicación es que las empresas que operan actualmente en la misma industria no compiten solo entre si sino respaldadas por sus propias cadenas de suministro. Ello significa que el conocimiento necesario para competir no puede almacenarse en una única empresa porque tiene que fluir a través de la red de empresas colaboradoras, incluidas las de la cadena de suministro. Las empresas con un mayor nivel de cooperación tecnológica externa pueden ofrecer mecanismos organizativos más fuertes de capacidad de absorción en términos de reconocimiento, comunicación y asimilación de conocimientos relevantes que les permitiría tener una comunicación bien establecida con otros agentes mediante procesos de intercambio de conocimientos. Este proceso de comunicación, a su vez, puede inspirar nuevas ideas para nuevos productos y procesos lo que implica no solo mejores resultados de innovación sino también una mayor ventaja competitiva en entornos ágiles. Si una mayor cooperación tecnológica es capaz de mejorar la eficacia y eficiencia de las Operaciones a través de la adopción y mejora de las nuevas tecnologías, entonces las empresas con mayor necesidad de flexibilidad de producción podrían beneficiarse más de los flujos externos de conocimiento y como consecuencia aumentar su capacidad de agilidad a través de los procesos de absorción del conocimiento.

En base a estos argumentos proponemos en este trabajo un efecto moderador de la cooperación externa en innovación sobre la relación entre la flexibilidad de producción y la capacidad de agilidad. La literatura evidencia que las tecnologías de producción flexible (robots, prototipado rápido, etc.) contribuyen a desarrollar la producción ágil (Narasimhan et al., 2006; Vázquez $\square$ Bustelo et al., 2007). Por ello las empresas con más acceso a los conocimientos externos de la cadena de suministro y otros agentes como institutos de investigación podrían desarrollar su agilidad a través de las mejoras en las tecnologías y procesos de producción que se consigan absorbiendo dicho conocimiento externo. La cooperación externa podría tener entonces un efecto moderador sobre la relación entre flexibilidad de producción y capacidad de agilidad. Las empresas con mayor flexibilidad de producción podrían desarrollar así una mayor capacidad de agilidad si cooperasen externamente más en innovación. También pudiera darse el caso de que la cooperación externa en innovación compensase los menores esfuerzos en desarrollar la flexibilidad de producción, de tal forma que las empresas con menor flexibilidad de producción podrían desarrollar así una mayor capacidad de agilidad si al menos cooperasen externamente más en innovación.

H1. La cooperación externa en innovación modera la relación entre la capacidad de flexibilidad de producción y la capacidad de agilidad de la empresa.

Aunque el acceso externo a nuevos conocimientos es importante para aumentar la capacidad de absorción de conocimientos, las empresas deben también desarrollar organizaciones flexibles que apoyen la contribución de los empleados a la innovación y faciliten el cambio requerido por las organizaciones ágiles. La producción ágil necesita un mayor acceso y aplicación de conocimientos que otros sistemas de producción porque tienen que innovar mejor y más rápido. Inicialmente tanto la innovación interna como la cooperación tecnológica externa pueden apoyar la asimilación y transformación de conocimiento externo en conocimiento interno para gestionar los sistemas de producción de una forma más competitiva y ágil. Sin embargo, la inversión en recursos no es suficiente si, por ejemplo, la organización es burocrática y ralentiza el proceso de toma de decisiones o desincentiva la adopción de riesgos. Por tanto, el apoyo organizativo a la innovación puede apalancar los esfuerzos de innovación de la empresa en una mayor capacidad de agilidad mediante la promoción de comportamientos innovadores facilitando la rápida toma de decisiones.

H2. El apoyo organizativo a la innovación modera la relación entre la capacidad de innovación de producto de la empresa y su capacidad de agilidad.

La Figura 1 presenta nuestro modelo de investigación con las hipótesis propuestas. La próxima sección explica la metodología del estudio empírico que contrasta dicho modelo. 
Figura 1 Modelo de investigación

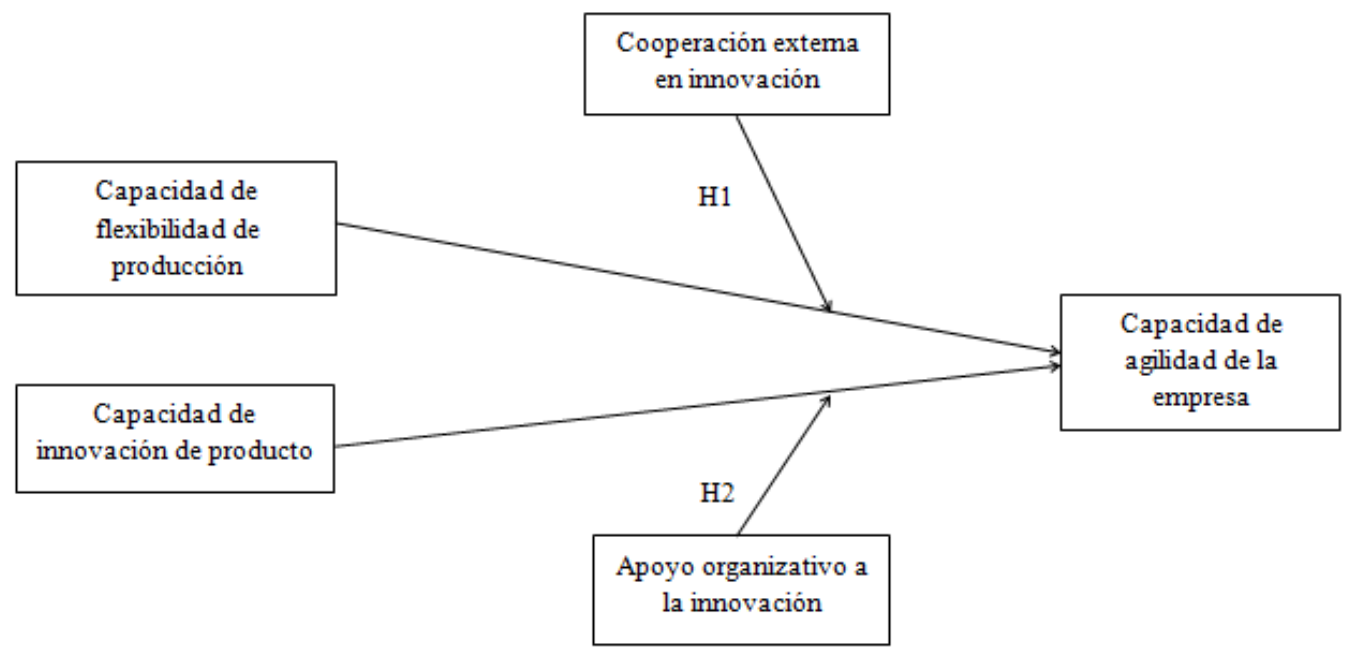

\section{Metodología}

La información para el estudio se ha obtenido de una encuesta por correo dirigida a Directores de Producción y de Recursos Humanos de empresas manufactureras españolas. El Director/Directora de Producción era quien contestaba todas las preguntas relacionadas con la producción ágil mientras que su colega de Recursos Humanos atendía las de flexibilidad, innovación y resultados. La población de empresas para el estudio se tomó de la base de datos SABI (Sistema de Balances de Análisis Ibéricos) en los CNAE 24 a 32 inclusive y con 200 empleados de plantilla como mínimo para asegurar la existencia de departamentos especializados de producción y recursos humanos. El número de empresas de la población ascendía a 619, de las cuales contestaron a la encuesta un total de 227 empresas (tabla 1) después de dos recordatorios, abarcando el periodo de recogida de datos desde Enero a Mayo de 2017. La tasa de respuesta (36\%) se considera suficientemente buena en comparación con otros estudios de la producción ágil y de acuerdo con los estándares establecidos por Baruch (1999) para encuestas dirigidas a directivos. El sesgo de no respuesta lo controlamos comprobando si los primeros y últimos cuestionarios recibidos diferían significativamente (Armstrong y Overton, 1977), no siendo este el caso para nuestro estudio según las pruebas de Mann-Whitney con las variables demográficas y los principales constructos.
Tabla 1 Distribución de las empresas de la muestra

\begin{tabular}{|c|c|c|}
\hline CNAE & $\mathrm{N}^{\mathrm{o}}$ empresas & $\%$ total \\
\hline 24. Metalurgia: fabricación de productos de hierro, acero y ferroaleaciones & 23 & 10,1 \\
\hline 25. Fabricación de productos metálicos, excepto maquinaria y equipo & 34 & 15,0 \\
\hline 26. Fabricación de productos informáticos, electrónicos y ópticos & 22 & 9,7 \\
\hline 27. Fabricación de material y equipo eléctrico & 23 & 10,1 \\
\hline 28. Fabricación de maquinaria y equipo & 29 & 12,8 \\
\hline 29. Fabricación de vehículos de motor, remolques y semirremolques & 56 & 24,7 \\
\hline 30. Fabricación de otro material de transporte & 19 & 8,4 \\
\hline 31. Fabricación de muebles & 12 & 5,3 \\
\hline 32. Otras industrias manufactureras & 9 & 4,0 \\
\hline Total empresas & 227 & 100,0 \\
\hline
\end{tabular}


Las variables utilizadas en nuestro trabajo empírico proceden mayormente de otros estudios que las han contrastado previamente. En primer lugar, para las prácticas de fabricación y suministro que facilitan la agilidad en el sistema de producción se han utilizado y adaptado las escalas de Narasimhan et al. (2006). Estas escalas valoran el grado de implantación de las distintas técnicas en la planta comparándolo con el promedio de la industria según una escala Likert de 1 ("mucho menos implantado en la planta que el promedio de la industria") a 7 ("mucho más implantado en la planta que el promedio de la industria"). Los constructos utilizados son los siguientes (entre paréntesis se indica el número de ítems procedentes del trabajo de Narasimhan et al. (2006) que comprende cada constructo): Desarrollo de empleados (8); Equipos de trabajo (5); Cultura de Calidad Total (4); Información compartida con proveedores (3); Desarrollo de proveedores (3); Asociación con proveedores (4); Racionalización de la base de proveedores (3); Selección estratégica de proveedores (3); Certificación de proveedores (2); MRP/ ERP avanzado (2); Diseño integrado de producto (7); Tecnologías avanzadas de fabricación (6); Desarrollo de tecnología de proceso (4); Flujo de materiales Justo a Tiempo (6); Fabricación en células (2); Ingeniería concurrente (4); Orientación a los clientes (7); Integración de la estrategia de fabricación (6); Benchmarking (2). Aunque no sea exactamente el objetivo de nuestro estudio, también contrastamos si las empresas con mayor nivel agilidad han implantado en mayor medida estás prácticas de fabricación y suministro.

La tabla 2 indica las variables utilizadas en el análisis de regresión jerárquica para contrastar las hipótesis del estudio, es decir el explicar el nivel de agilidad de la empresa sobre la base de sus capacidades de innovación y de producción con los dos efectos moderadores de la cooperación externa en innovación y del apoyo organizativo a la innovación. Para relativizar la contribución de las variables independientes se han utilizado dos variables de control -tamaño de empresa $\left(\mathrm{n}^{\mathrm{o}}\right.$ de empleados) y propensión exportadora (porcentaje de la facturación exportado)- y dos determinantes o drivers de la agilidad: dinamismo del entorno (constructo de 8 ítems) y hostilidad del mercado (constructo de 3 ítems). Efectuamos un análisis factorial exploratorio (rotación Varimax) para comprobar la unidimensionalidad de los constructos: el porcentaje de varianza explicada (VE) superaba el 50\% y todos los ítems tenían un factor superior a 0,5. Todos los constructos tienen además un alfa de Cronbach $(\alpha)$ superior a 0,7 lo que se considera adecuado. Adicionalmente los índices de fiabilidad compuesta de cada constructo (IFC) dan valores superiores al 0,6 recomendado. La tabla 2 recoge todos estos indicadores para cada constructo con sus fuentes respectivas si procede, mientras que la tabla 3 muestra las estadísticas descriptivas de las variables utilizadas en el análisis de regresión.
Tabla 2 Descripción de las variables del estudio empírico

Notas.- VE $=$ Varianza Extraída; $\alpha$

= alfa de Cronbach: IFC = Índice de Fiabilidad Compuesta
Capacidad de agilidad.- VE $=83 \%, \alpha=0,966$, IFC=0,93. 7 ítems de Zhang (2011) con escala Likert de 1 "muy poco importante" a 7 "extremadamente importante".

Capacidad de flexibilidad de producción.- $\mathrm{VE}=80 \%, \alpha=0,918, \mathrm{IFC}=0,89.4$ ítems con escala Likert de 1 "totalmente en desacuerdo" a 7 "totalmente de acuerdo".

Capacidad de innovación de productos.- $\mathrm{VE}=76 \%, \alpha=0,881, \mathrm{IFC}=0,85.5$ ítems con escala Likert de 1 "totalmente en desacuerdo" a 7 "totalmente de acuerdo".

Cooperación externa en innovación.- $\mathrm{VE}=78 \%, \alpha=0,952$, IFC $=0,93.7$ ítems con escala Likert de 1 "extremadamente inferior al promedio de la industria" a 7 "extremadamente superior al promedio de la industria".

Apoyo organizativo a la innovación.- $\mathrm{VE}=84 \%, \alpha=0,938, \mathrm{IFC}=0,92.4$ ítems de Verdú-Jover et al. (2005) con escala Likert de 1 "totalmente en desacuerdo" a 7 "totalmente de acuerdo".

Dinamismo del entorno.- $\mathrm{VE}=80 \%, \alpha=0,967, \mathrm{IFC}=0,87.8$ ítems de Klaas et al. (2001) con escala Likert de 1 "totalmente en desacuerdo" a 7 "totalmente de acuerdo".

Hostilidad del mercado.- $\mathrm{VE}=85 \%, \alpha=0,910, \mathrm{IFC}=0,84.3$ ítems con escala Likert de 1 "totalmente en desacuerdo" a 7 "totalmente de acuerdo". 
Tabla 3 Estadísticas descriptivas (medias, desviaciones estándar y correlaciones)

$+\mathrm{p}<0,1 * \mathrm{p}<0,05 * * \mathrm{p}<0,01$

\begin{tabular}{|c|c|c|c|c|c|c|c|c|c|c|}
\hline & Mean & S.D. & 1 & 2 & 3 & 4 & 5 & 6 & 7 & 8 \\
\hline $\begin{array}{l}\text { 1.Capaci- } \\
\text { dad de agi- } \\
\text { lidad }\end{array}$ & 4,13 & 1,98 & & & & & & & & \\
\hline $\begin{array}{l}\text { 2.Tamaño } \\
\text { de empresa }\end{array}$ & 380 & 185 &,- 062 & & & & & & & \\
\hline $\begin{array}{l}\text { 3.Propen- } \\
\text { sión expor- } \\
\text { tadora }\end{array}$ & 29,3 & 23,5 & ,408* &,- 053 & & & & & & \\
\hline $\begin{array}{l}\text { 4.Dinamis- } \\
\text { mo }\end{array}$ & 3,96 & 2,01 &, $557 * *$ &,- 053 & ,245+ & & & & & \\
\hline $\begin{array}{l}\text { 5. H o st i - } \\
\text { lidad del } \\
\text { mercado }\end{array}$ & 4,02 & 1,96 & ,378* &,- 054 & ,218+ &, $362^{*}$ & & & & \\
\hline $\begin{array}{l}\text { 6.Flexibili- } \\
\text { dad de pro- } \\
\text { ducción }\end{array}$ & 4,07 & 2,00 & ,486* &,- 049 &, $500 *$ &, $559 * *$ &, $478^{* *}$ & & & \\
\hline $\begin{array}{l}7 \text {. I n n o - } \\
\text { vación de } \\
\text { producto }\end{array}$ & 4,28 & 1,39 &, $650 * *$ & ,118 &, $465^{*}$ &, $527^{*}$ &, $535^{* *}$ & ,458* & & \\
\hline $\begin{array}{l}\text { 8.Cooper- } \\
\text { ación ex- } \\
\text { terna }\end{array}$ & 3,42 & 1,65 &, $444 * *$ & 160 &, $527^{*}$ & 116, &, $325^{*}$ &, $517 * *$ &, $589 * *$ & \\
\hline $\begin{array}{l}\text { 9.Apoyo or- } \\
\text { ganizativo }\end{array}$ & 3,59 & 1,41 & $686^{* *}$ &,- 076 & ,132 &, $357^{*}$ &, $213+$ &, $425 * *$ &, $633 * *$ &, $541 * *$ \\
\hline
\end{tabular}

\section{Resultados}

En primer lugar, la tabla 4 muestra las diferencias de medias en la implantación de técnicas de gestión de fabricación y suministro según el grado de capacidad de agilidad de las empresas manufactureras. Los resultados indican que las empresas con mayor agilidad las han implantado en mayor medida que las empresas menos ágiles. Estas técnicas están presentes en la mayor parte de las empresas pero la diferencia estriba en el grado o intensidad de dicha implantación que es mucho mayor para aquellas empresas que necesitan una mayor capacidad de agilidad. Agregándolas por valor de importancia, pueden observarse tres grupos: el correspondiente a la gestión de la cadena de suministro (en lo referente a integración de información con MRP/ERP y la certificación y selección de proveedores), el del potenciamiento de los recursos humanos de la empresa (por ejemplo, el desarrollo de empleados o la calidad total) y el de algunas tecnologías de fabricación ágil (como la ingeniería concurrente y las células de fabricación). La Figura 2 (anexo) muestra un modelo de factores de gestión de la producción ágil en base a estos resultados

En segundo lugar, la tabla 5 evidencia que las empresas más agiles han desarrollado también más otras capacidades de gestión que las menos ágiles: producción, innovación de producto y cooperación externa en innovación. Destaca que la mayor capacidad de gestión y donde existe más diferencia según el nivel de agilidad es en el apoyo organizativo a la innovación. Es decir, para conseguir agilidad las empresas manufactureras españolas están prestando atención en primer lugar al apoyo organizativo a la innovación, seguida de las capacidades de producción (flexibilidad y fabricación) y de las capacidades de innovación interna y externa. 
Tabla 4 Diferencias de implantación de técnicas de fabricación y suministro en las empresas manufactureras españolas, según el nivel de capacidad de agilidad

Notas.- Nivel de significación del contraste de medias $* * \mathrm{p}<0,01$ Los constructos son promedios de la valoración de los ítems que los componen y que están valorados en escala Likert de 1 (mucho menos implantado en la planta que en el promedio de la industria) a 7 (mucho más implantado en la planta que en el promedio de la industria). Las empresas se han clasificado entre baja y alta agilidad según el valor de la variable Agilidad que representa la capacidad de agilidad requerida por los cambios en el entorno de negocio; se ha tomado el valor medio de 4,13 para separar a las empresas en dos grupos: baja agilidad $(<4,13)$ y alta agilidad $(\geq 4,13)$
Constructos de técnicas de fabricación y suministro

Empresas con alta agilidad $(\mathrm{n}=121)$

Empresas con baja agilidad $(\mathrm{n}=106)$ que facilitan agilidad

\begin{tabular}{|c|c|c|}
\hline MRP/ERP avanzado & $6,44^{* *}$ & 3,95 \\
\hline Certificación de proveedores & $6,07 * *$ & 4,26 \\
\hline Selección estratégica de proveedores & $5,76^{* *}$ & 2,34 \\
\hline Diseño integrado de producto & $5,76^{* *}$ & 2,28 \\
\hline Desarrollo de tecnología de proceso & $5,75^{* *}$ & 2,33 \\
\hline Tecnologías avanzadas de fabricación & $5,73^{* *}$ & 2,20 \\
\hline Desarrollo de empleados & $5,71 * *$ & 2,19 \\
\hline Benchmarking & $5,70 * *$ & 2,46 \\
\hline Cultura de Calidad Total & $5,69 * *$ & 2,35 \\
\hline Racionalización de la base de proveedores & $5,68 * *$ & 2,45 \\
\hline Flujo de materiales Justo a Tiempo & $5,67 * *$ & 2,28 \\
\hline Información compartida con proveedores & $5,66^{* *}$ & 2,28 \\
\hline Asociación con proveedores & $5,63 * *$ & 2,32 \\
\hline Ingeniería concurrente & $5,61^{* *}$ & 2,20 \\
\hline Orientación a los clientes & $5,60 * *$ & 2,26 \\
\hline Fabricación en células & $5,59 * *$ & 2,12 \\
\hline Integración de la estrategia de fabricación & $5,56 * *$ & 2,40 \\
\hline Desarrollo de proveedores & $5,48 * *$ & 2,15 \\
\hline Equipos de trabajo & $5,44 * *$ & 2,13 \\
\hline
\end{tabular}


Tabla 5 Diferencias de capacidades de gestión en las empresas manufactureras españolas, según el nivel de agilidad

Notas.- Nivel de significación del contraste de medias $* * \mathrm{p}<0,01$.

Las empresas se han clasificado entre baja y alta agilidad según el valor de la variable Agilidad que representa la capacidad de agilidad requerida por los cambios en el entorno de negocio; se ha tomado el valor medio de 4,13 para separar a las empresas en dos grupos: baja agilidad $(<4,13)$ y alta agilidad $(\geq 4,13)$.
Empresas con alta agilidad $(\mathrm{n}=121)$

Empresas con baja agilidad $(\mathrm{n}=106)$

\begin{tabular}{lcc} 
Apoyo organizativo a la innovación & $5,77^{* *}$ & 2,18 \\
Capacidad de flexibilidad de producción & $5,68^{* *}$ & 2,23 \\
Capacidad de gestión de fabricación & $5,68^{* *}$ & 2,23 \\
Capacidad de innovación de productos & $5,66^{* *}$ & 2,26 \\
& & 1,89 \\
\hline
\end{tabular}

Por último, la tabla 6 muestra el análisis estadístico de regresión jerárquica que explica la agilidad de las empresas con los efectos moderadores de la cooperación externa en innovación y el apoyo organizativo a la innovación. La primera parte de la regresión del Modelo 3 indica que la única variable significativa es el driver de la hostilidad del mercado: las empresas más agiles se enfrentan a mercados más hostiles $(\beta=0,168, p<0,01)$; la propensión exportadora (variable de control) solo está marginalmente relacionada con la capacidad de agilidad. En relación con las variables independientes que explican la capacidad de agilidad, tanto la capacidad de flexibilidad de producción $(\beta=0,359, \mathrm{p}<0,01)$ como la capacidad de innovación de producto $(\beta=0,074, \mathrm{p}<0,05)$ están positiva y significativamente relacionadas con la agilidad. La última parte de la regresión incluye los efectos moderadores de la cooperación externa y el apoyo organizativo a la innovación. Los resultados indican que ambas variables moderadoras están positivamente relacionados con la capacidad de agilidad: cooperación externa $(\beta=0,224, \mathrm{p}<0,01)$ y apoyo organizativo $(\beta=0,416, p<0,01)$; las empresas más ágiles cooperan externamente y apoyan internamente más a la innovación que las empresas menos ágiles. Sin embargo, solo la cooperación externa es un moderador significativo $(\beta=-0,204, p<0,05)$ lo cual apoya la hipótesis $\mathrm{H} 1$ pero no valida la hipótesis $\mathrm{H} 2$.
Tabla 6 Análisis de regresión lineal de la capacidad de agilidad en empresas manufactureras

Notas.- Coeficientes $\beta$ estandarizados. Valores de t-student entre paréntesis. Niveles de significación $+\mathrm{p}<0,1$ $* * \mathrm{p}<0,01$

\begin{tabular}{|c|c|c|c|}
\hline & Modelo 1 & Modelo 2 & Modelo 3 \\
\hline Tamaño de empresa ( $n^{\circ}$ empleados) & $-0,009(0,66)$ & $0,013(1,28)$ & $0,004(0,55)$ \\
\hline Propensión exportadora (\% exportaciones/facturación) & $0,014(0,61)$ & $0,021(1,19)$ & $0,028+(1,93)$ \\
\hline Dinamismo del entorno & $0,764 * *(14,76)$ & $0,044(1,02)$ & $0,015(0,49)$ \\
\hline Hostilidad del mercado & $0,210^{* *}(4,25)$ & $0,287 * *(5,12)$ & $0,168 * *(3,97)$ \\
\hline Capacidad de flexibilidad de producción (CFP) & - & $0,577 * *(9,21)$ & $0,359^{* *}(6,25)$ \\
\hline Capacidad de innovación de producto (CIP) & - & $0,071 *(2,01)$ & $0,074 *(2,08)$ \\
\hline Cooperación externa en innovación (CEI) & - & - & $0,224 * *(3,23)$ \\
\hline CFP x CEI & - & - & $-0,204 *(2,15)$ \\
\hline Apoyo organizativo a la innovación (AOI) & - & - & $0,416^{* *}(5,82)$ \\
\hline CIP x AOI & - & - & $0,012(0,12)$ \\
\hline \multirow[t]{3}{*}{ Estadísticas del modelo } & $\mathrm{F}=1340,92 * *$ & $\mathrm{~F}=1543,04 * *$ & $\mathrm{~F}=1796,57 * *$ \\
\hline & $\mathrm{R} 2$ ajustado=0,58 & R2 ajustado=0,69 & R2 ajustado $=0,81$ \\
\hline & & Cambio R2=0,11 & Cambio R2 $=0,12$ \\
\hline
\end{tabular}




\section{Implicaciones para las empresas}

Los resultados cuantitativos de nuestro estudio corroboran las conclusiones de otros trabajos sobre la importancia de la tecnología en las capacidades de agilidad pero también aportan elementos diferenciadores para su gestión. Así por ejemplo, se ha puesto de manifiesto que en las empresas analizadas se presta más importancia a la cadena de suministro, los recursos humanos y las tecnologías de fabricación (tabla 4). De este resultado destaca la importancia otorgada a los recursos humanos, tanto en sí misma como por la relación que tiene con los otros dos bloques de la Figura 2. La agilidad no puede alcanzarse sin apalancarse sobre las habilidades y el conocimiento de los empleados. La agilidad de los recursos humanos necesita que la innovación aumente la base de habilidades de los empleados por encima de las necesidades inmediatas. Las empresas que desean ser agiles deberían centrarse no solo en la tecnología sino en formar a los trabajadores en el uso de dicha tecnología. Un clima efectivo de formación obliga a la organización a crear y mantener un entorno de aprendizaje en el que se estimule a las personas a ser más abiertas e innovadoras en la búsqueda de nuevas ideas. Ello redundará en facilitar la cooperación técnica con otras empresas y la adquisición de conocimientos y habilidades de aprendizaje, y como consecuencia en la capacidad para adaptarse y responder a los cambios en el mercado y en los entornos laborales.

En cuanto a la explicación de la capacidad de agilidad de las empresas, nuestro estudio ha evidenciado asimismo algunos resultados singulares. En primer lugar, hemos encontrado que el apoyo organizativo a la innovación está relacionado positivamente con la agilidad (tabla 6) y dicha variable de apoyo organizativo es la que muestra mayor diferencia de medias entre las empresas más ágiles y las menos ágiles (tabla 5). El apoyo organizativo a la innovación es un intangible de la empresa que contribuye a uno de los pilares de la producción ágil, concretamente la de la cadena de valor innovadora intensiva en conocimiento. El diseño de una cadena de valor que responda a mercados donde se demandan productos muy personalizados con plazos reducidos de entrega es algo muy crítico. En este contexto no solo es necesaria una elevada capacidad de flexibilidad de fabricación sino también una interacción fluida entre marketing, diseño de producto y fabricación personalizada. Dichas interacciones precisan de un adecuado apoyo organizativo que sirva de puente para agilizar los procesos. Este apoyo organizativo puede facilitar en concreto algunas de las técnicas utilizadas para gestionar cadenas de valor como, por ejemplo, los sistemas ERP basados en Internet (Elmoselhy, 2013). Las empresas están desarrollando cadenas de valor electrónicas en las que las Redes de Área Local (LAN) de las máquinas de cada empresa de la cadena de valor están conectadas entre sí a través de Internet y un servidor de la oficina central que recoge la información existente en tarjetas electrónicas identificativas y transmitida por tecnologías de identificación de radio frecuencia (RFID).
Otro resultado y contribución singular de nuestra investigación es el efecto moderador de la cooperación externa. La literatura reconoce los efectos positivos de la cooperación para compartir los costes de innovación y reducir el tiempo de desarrollo de las innovaciones de producto. La cooperación inter-organizativa en innovación ha sido utilizada como moderador en estudios de flexibilidad de recursos humanos para diferenciar la contribución de cada dimensión de flexibilidad (por ejemplo, Martínez-Sánchez et al., 2011). Nuestro estudio es la primera contrastación empírica de este efecto moderador aplicado a otra importante dimensión de flexibilidad, cual es la flexibilidad de producción. Los resultados de la tabla 6 indican que la cooperación externa en innovación modera negativamente la relación entre capacidad de flexibilidad de producción y capacidad de agilidad $(\beta=-0,204, p<0,05)$. Este efecto moderador negativo sugiere que las empresas con menor flexibilidad de producción pueden también ser más ágiles si en cambio cooperan externamente más en innovación. El acceso al conocimiento externo y las mejores prácticas existentes fuera de la empresa pueden complementar los esfuerzos internos para desarrollar capacidades de flexibilidad de producción. Las empresas no necesitan poseer todos los recursos necesarios para competir; en algunos recursos es suficiente con que la empresa los controle o tenga la oportunidad de acceder a ellos de forma temporal.

La ausencia de efecto moderador del apoyo organizativo a la innovación en la relación entre capacidad de innovación de producto y la capacidad de agilidad (tabla 6), nos indica que esta relación es independiente del nivel de apoyo organizativo de la empresa hacia la innovación. Este resultado puede venir condicionado por el hecho, ya comentado, de que el apoyo organizativo a la innovación es la capacidad que presenta valores más altos en las empresas encuestadas (tabla 5) aunque bien es verdad que también muestra mayor diferencia de medias entre empresas más ágiles y menos ágiles. Las empresas ágiles han de innovar más y más rápido que las demás por lo que el apoyo organizativo a la innovación debe existir como un requisito previo y no condicionado si quieren enfrentarse con éxito a cambios continuos e impredecibles en las condiciones del mercado y las necesidades de los clientes. La importancia otorgada a las prácticas de recursos humanos tanto en sí mismas como elemento también necesario para el funcionamiento de otras prácticas más relacionadas con la producción y la cadena de suministro, hace que el esfuerzo de innovación interno vaya acompañado necesariamente de aquellos intangibles que, como el apoyo organizativo, permitan desarrollar al máximo las capacidades necesarias para absorber conocimientos y transformarlos en innovaciones de producto. La existencia del efecto moderador de la cooperación externa junto con la ausencia del efecto moderador del apoyo organizativo interno pone el acento en el acceso a las mejores prácticas y conocimiento del exterior para la transformación de las capacidades internas de producción en capacidades de agilidad empresarial. 


\section{Conclusión y limitaciones del estudio}

Nuestro estudio ha evidenciado que las empresas manufactureras con mayor capacidad de agilidad son las que han implantado en mayor medida todo un conjunto de técnicas de gestión de fabricación y suministro. Simplificadamente puede considerarse que para la producción ágil de las empresas manufactureras españolas lo más importante es el desarrollo del conocimiento y habilidades de los recursos humanos, la gestión ágil de la cadena de suministro y la implantación de tecnologías ágiles de fabricación. Pero la principal conclusión y objetivo de nuestro trabajo ha sido constatar que las empresas manufactureras más ágiles cooperan externamente más en innovación y que dicha cooperación modera además la flexibilidad de producción de las empresas, indicando con ello que, gracias a la cooperación externa en innovación, las empresas con menor flexibilidad de producción pueden aun así conseguir mayor capacidad de agilidad. No hemos obtenido un efecto moderador del apoyo organizativo a la innovación pero sí que dicho apoyo es la capacidad más desarrollada y con una mayor diferencia según el nivel de agilidad de las empresas, lo que refuerza la importancia de los recursos humanos en el desarrollo de los sistemas de producción ágil.

Las principales limitaciones de nuestro diseño de investigación es el uso de datos de corte transversal y con percepciones directivas para evaluar la mayoría de las variables. Futuros trabajos podrían usar datos longitudinales para confirmar las relaciones apuntadas en este estudio. También reconocemos la dificultad de generalizar nuestros resultados que están basados únicamente en empresas españolas, por lo que una base de datos con más empresas europeas serviría también para contrastar nuestro modelo y propuestas.

Agradecimientos.- La investigación en la que está basada este trabajo ha sido financiada con el proyecto ECO201456912-R del Programa Estatal de I+D+i.

\section{Referencias}

1. Amstrong, J. y Overton, T. (1977), "Estimating nonresponse bias in mail surveys", Journal of Marketing Research, Vol. 14 No. 3, pp. 396-402.

2. Barney, J. (1991), "Firm resources and sustained competitive advantage", Journal of Management, Vol. 17 No. 1, pp. 99-120.

3. Baruch, Y. (1999), "Response rate in academic studies a comparative analysis”, Human Relations, Vol. 52 No. 4, pp. 421-438.
4. Dobrzykowski, D., Leuschner, R. y Hong, P. (2015), "Examining absorptive capacity in supply chains: Linking responsive strategy and firm performance", Journal of Supply Chain Management, Vol. 51 No. 4, pp. 3-28.

5. Dubey, R. y Gunasekaran, A. (2015), “Agile manufacturing: framework and its empirical validation", International Journal of Advance Manufacturing Technology, Vol. 76, pp. 2147-2157.

6. Cohen, W. y Levinthal, D. (1990), “Absorptive capacity: A new perspective on learning and innovation”, Administrative Science Quarterly, Vol.35 No. 1, pp. 128-152.

7. Elmoselhy, S.A.M (2013), "Hybrid lean-agile manufacturing system technical facet in automotive sector", Journal of Manufacturing Systems, Vol. 32, pp. 598619.

8. Goldman, S.L. y Nagel, R.N. (1991). 21st Century Manufacturing Enterprise Strategy: An Industry-Led View. Iacocca Institute, Lehigh University, Beghlehem, PA.

9. Gligor, D., Esmark, C. y Holcomb, M. (2015). "Performances outcomes of supply chain agility: When should you be agile?”. Journal of Operations Management, Vol. 33 No 1, pp. 71-82.

10. Gunasekaran, A. y Yusuf, Y.Y. (2002), “Agile manufacturing: a taxonomy of strategic and technological imperatives”, International Journal of Production Research, Vol. 40 No. 6, pp. 1357-1385.

11. Klaas, B., McClendon, J. y Gainey, T. (2001), “Outsourcing HR: the impact of organizational characteristics”, Human Resource Management, Vol. 40, pp. 125138.

12. Kohlbacher, M. (2013), "The impact of dynamic capabilities through continuous improvement on innovation: The role of business process orientation”, Knowledge and Process Management, Vol. 20 No. 2, pp. 71-76.

13. Lee, H. (2004), "The triple A supply chain", Harvard Business Review, Vol. 82 No. 10, pp. 102-112.

14. Martínez, A. y Lahoz, F. (2017), "La agilidad en la cadena de suministro y la capacidad de absorción de conocimientos - Influencia en los resultados empresariales", Economía Industrial, No. 403, pp. 135-142.

15. Martinez-Sánchez, A., Vela-Jimenez, M-J., Pérez-Pérez, M. y de-Luis-Carnicer, P. (2011), "The Dynamics of Labour Flexibility: Relationships between Employment Type and Innovativeness”, Journal of Management Studies, Vol. 48 No. 4, pp. 715-736. 
16. Narasimhan, R., Swink, M. y Kim S. (2006), "Disentangling leanness and agility: An empirical investigation”, Journal of Operations Management, Vol. 24, pp. 440457.

17. Teece, D., Pisano, G. y Shuen, A. (1997), "Dynamic capabilities and strategic management”, Strategic Management Journal, Vol. 18 No. 7, pp. 509-533.

18. Teece, D. (2007), "Explicating dynamic capabilities: the nature and microfoundations of (sustainable) enterprise performance”, Strategic Management Journal, Vol. 28 No. 13, pp. 1319-1350.

19. Vázquez-Bustelo, D., Avella, L. y Fernández, E. (2007), "Agility drivers, enablers and outcomes: Empirical test of an integrated agile manufacturing model”, International Journal of Operations \& Production Management, Vol. 27 No. 12, pp. 1303-1333.
20. Verdú-Jover, A., Llorens-Montes, J. y Garcia-Morales, V. (2005), "Flexibility, fit and innovative capacity: an empirical examination”, International Journal of Technology Management, Vol. 30, pp. 131-146.

21. Zahra, S. y George, G. (2002), “Absorptive capacity: A review, reconceptualization and extension”, Academy of Management Review, Vol. 27 No. 2, pp. 185-203.

22. Zhang, D.Z. (2011), "Towards theory building in agile manufacturing strategies - Case studies of an agility taxonomy", International Journal of Production Economics, Vol. 131, pp. 303-312.

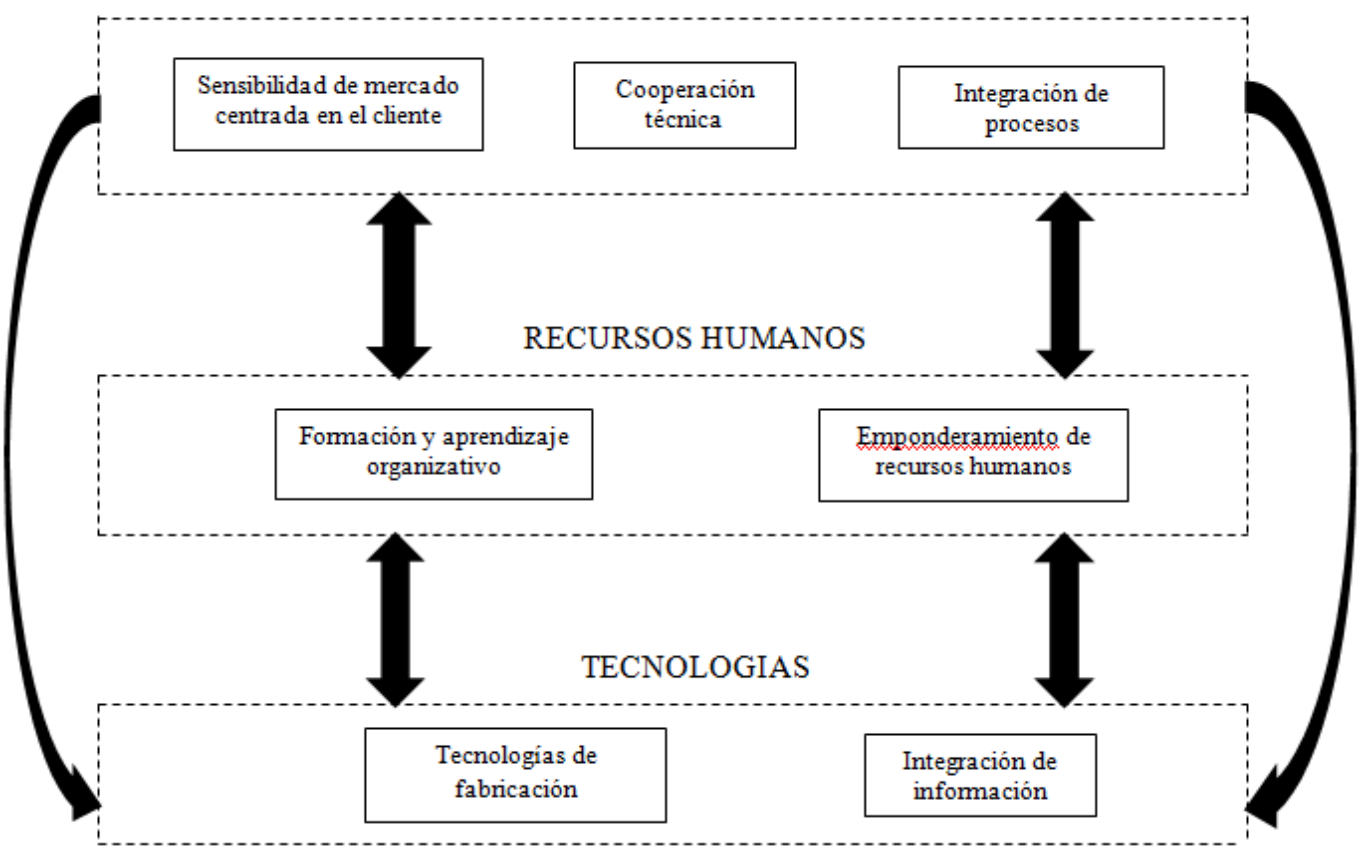

